\title{
Neighbourhood socioeconomic disadvantage and behavioural problems from late childhood into early adolescence
}

\author{
J Schneiders, M Drukker, J van der Ende, F C Verhulst, J van Os, N A Nicolson
}

J Epidemiol Community Health 2003;57:699-703

See end of article for authors' affiliations

.......................

Correspondence to: Dr N Nicolson, Department of Psychiatry and Neuropsychology (PAR45), Maastricht University PO Box 616, 6200 MD Maastricht, Netherlands; n.nicolson@sp.unimaas.n

Accepted for publication 26 March 2003
Study objective: This study investigates whether neighbourhood socioeconomic disadvantage may contribute to child behavioural and emotional problems, beyond the effects of parental socioeconomic status. It also examines the influence of neighbourhood disadvantage on changes in the frequency of behavioural problems from late childhood into early adolescence.

Design and setting: The study was conducted in a large community sample in Rotterdam, the Netherlands. An index of neighbourhood socioeconomic disadvantage was calculated for each of the city's 74 neighbourhoods. Multilevel regression analysis estimated effects of neighbourhood disadvantage and individual variables (parental socioeconomic status, child's gender, and age) on behavioural problems reported by children (Youth Self-Report) and parents (Child Behavior Checklist) and on changes in these scores over a two year follow up.

Participants: A cohort of all children born in 1978 and living in Rotterdam. Of those eligible, 73\% $(n=2587)$ participated in the first measurement (T1), at 10-12 years; $71 \%$ of the $T 1$ respondents participated again two years later (T2), at 12-14 years.

Main results: Neighbourhood disadvantage was associated with higher Total, Internalising, and Externalising Problems, as assessed with both the Child Behavior Checklist and the Youth Self-Report, even after controlling for parental socioeconomic status. Neighbourhood disadvantage also seemed to contribute to increases in Total Problems over the follow up.

Conclusions: Living in a disadvantaged neighbourhood is associated with greater behavioural problems and may lead to an exacerbation of problems as children move from childhood into adolescence. Public health interventions to improve child mental health must take the neighbourhood environment into account.
G rowing evidence indicates that neighbourhood socioeconomic disadvantage (NSD) influences many aspects of children's behaviour, ${ }^{1-6}$ but study designs and analytical techniques have hampered interpretation and generalisation of the results. Firstly, not all studies clearly distinguish effects of neighbourhood context from effects of individual or family level variables. The nesting of families within neighbourhoods necessitates an analytical approach that takes the hierarchical structure of the dataset into account. $^{7}$ Secondly, few studies examining neighbourhood effects have focused on the transition from childhood to

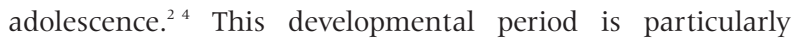
important: onset of behavioural problems in late childhood is a major risk factor for adult disorders, ${ }^{13}$ and the neighbourhood exerts new forms of influence as children become increasingly independent outside the home and have more contacts with neighbourhood peer groups, adults, and institutions. ${ }^{3}$ Finally, longitudinal studies of neighbourhood effects on mental health outcomes are scarce. If NSD plays a causal part, we would expect children living in disadvantaged neighbourhoods to show increasing problems as they get older. Follow up data are necessary to investigate this hypothesis.

The main goal of this study was to assess the independent impact of NSD on behavioural and emotional problems during the transition from late childhood into early adolescence. Data were collected during an earlier investigation ${ }^{8}$ of children born in 1978 in Rotterdam, the Netherlands, a large metropolitan area with over half a million residents. We hypothesised (1) that children's behavioural problems would be associated with neighbourhood disadvantage, over and above the effect of family socioeconomic status (SES), and (2) that NSD would contribute to an increase in behavioural problems over time. We also investigated whether the effects of living in a disadvantaged neighbourhood were different for children of high compared with low SES families, as has been previously reported.

\section{METHODS}

Setting, subjects, and procedure

Rotterdam has 74 residential neighbourhoods, as defined by the Dutch Central Bureau of Statistics. In 1988, these neighbourhoods ranged in size from 292 to 30990 inhabitants (median 7570), unemployment ranged from 3\% to $43 \%$ and welfare recipients from $0 \%$ to $21 \%$.

In the school year 1989-1990 (T1), children born in 1978 and living in Rotterdam were asked to take part in a study of behavioural problems in relation to pubertal development. ${ }^{810}$ A routine medical examination of all schoolchildren allowed access to the entire cohort. Mentally retarded children and children with non-Dutch nationality were excluded because of insufficient reading skills of the child or parent.

At $\mathrm{T} 1$ and two years later at $\mathrm{T} 2$, questionnaires were mailed to each child's parents. Children completed questionnaires at the medical examination (T1) or in the classroom (T2). Parental consent was obtained on both occasions. The study was

Abbreviations: NSD, neighbourhood socioeconomic disadvantage; SES, socioeconomic status; CBCL, Child Behavior Checklist; YSR, Youth Self-Report 
Table 1 Problem scores at baseline (T1) and follow up (T2)

\begin{tabular}{lcc}
\hline Problem score & T1 Mean (SD) & T2 Mean (SD) \\
\hline CBCL & & \\
Total & $20.42(16.42)$ & $18.78(16.07)$ \\
Internalising & $5.94(5.70)$ & $5.68(5.84)$ \\
$\quad$ Externalising & $7.20(6.90)$ & $6.73(6.73)$ \\
YSR & & \\
$\quad$ Total & $25.46(19.05)$ & $32.35(18.02)$ \\
Internalising & $7.80(6.64)$ & $9.63(7.24)$ \\
Externalising & $7.59(6.25)$ & $10.24(6.21)$ \\
\hline CBCL, Child Behavior Checklist; YSR, Youth Self-Report. At T1, \\
$\mathrm{n}=2496$ for CBCL measures; $\mathrm{n}=2329$ for YSR measures. At T2, \\
numbers were 1565 and 1787, respectively.
\end{tabular}

approved by the medical ethics committee of the University Hospital Rotterdam and conformed to the principles outlined in the Declaration of Helsinki.

\section{Measures}

Parent and self reported problems

We used the validated Dutch translations of the Child Behavior Checklist $\left(\mathrm{CBCL}^{11}\right)$ and the Youth Self-Report $\left(\mathrm{YSR}^{12}\right)$ to obtain information about children's problems from two perspectives. CBCL and YSR measures generally display only moderate correlations, with decreasing agreement as children get older. ${ }^{13}$

The parent completed CBCL includes 120 problems, each rated on a 3 point scale $(0=$ not true, $1=$ sometimes true, $2=$ often true). A Total Problems score is computed by summing 118 items. Scores were also calculated for the two syndrome dimensions Internalising (Withdrawn, Somatic Complaints, and Anxious/Depressed subscales) and Externalising behaviour (Delinquent and Aggressive subscales).

The child completed YSR, based on the CBCL, includes 103 behavioural and emotional problems, rated on 3 point scales, as above. Total Problems, Internalising and Externalising scores were calculated.

\section{Family SES}

Parental education and current occupation (for two parent families, the highest level) were assessed at T1, following validated procedures. Parental education and occupation were measured on ordinal 6 point scales, from uncompleted elementary school to postgraduate education and from no job/unskilled labour to higher professional status, respectively. The scales were moderately correlated $(r=0.63, \mathrm{n}=3331$, $\mathrm{p}<0.001)$.

\section{Neighbourhood socioeconomic disadvantage}

The Rotterdam City Bureau of Research and Statistics provided a summary measure of NSD, based on administrative data and derived through principal components analysis of eight indices of disadvantage aggregated at the neighbourhood level: percentage 17 year olds enrolled in education; percentage adults ( $\geqslant 20$ years) receiving welfare; percentage residents with non-Dutch nationality or born in Surinam or the Dutch Antilles; percentage adult male unemployment; percentage residents moving out of the neighbourhood in a one year period; percentage households with married couples and/or children; mean income; and mean age of residential buildings. NSD score was standardised, with Z scores for individual neighbourhoods ranging from -1.98 (relative advantage) to 1.69 (relative disadvantage).

\section{Statistical analysis}

Statistical tests were two tailed $(\alpha=0.05)$. MLWIN (Institute of Education, London) was used for estimating multilevel models. Multilevel regression analysis is ideally suited for

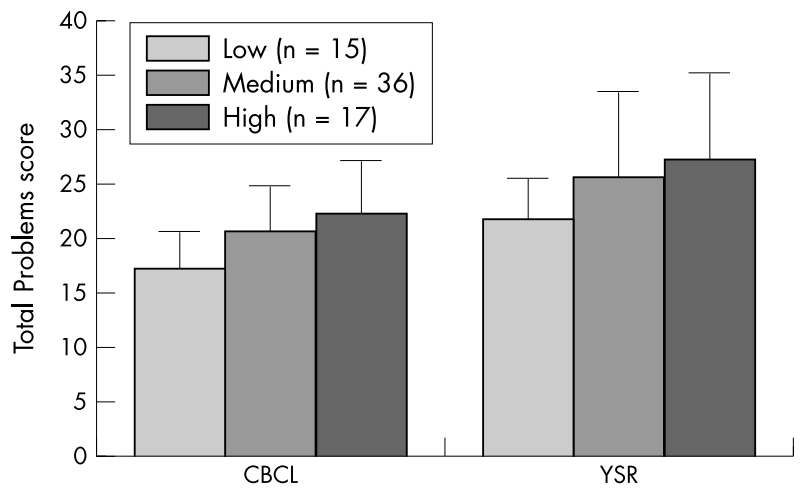

Figure 1 Total T1 Problem scores and level of neighbourhood disadvantage (NSD). Mean problem scores were first calculated per neighbourhood. Cut off points for low/high NSD were 1 SD below/above the mean level. Error bars represent standard deviations.

hierarchically clustered data. ${ }^{7}$ The model used to estimate neighbourhood effects on children's behaviour had three levels: because participants were sampled at $\mathrm{Tl}$ and $\mathrm{T} 2$, assessments (level 1 ) were clustered within individuals (level 2), who were clustered within neighbourhoods (level 3). Level I variables included the dependent CBCL and YSR measures and child age at assessment. Level 2 predictors were child gender and parental SES at Tl. NSD was a level 3 predictor.

After estimating an initial model with NSD as the only predictor of child problems, we extended the model to control for family SES variables parental occupation (OCC) and education (EDUC), child AGE, and GENDER $(0=$ male, $1=$ female $)$. In the model specified below, intercepts were allowed to vary randomly across neighbourhoods, individuals, and measurements, while slopes were modelled as fixed:

$$
\begin{aligned}
& \text { problem score }=\beta_{0}+\beta_{1} \mathrm{NSD}_{\mathrm{k}}+\beta_{2} \mathrm{OCC}_{\mathrm{ik}}+\beta_{3} \mathrm{EDUC}_{\mathrm{ik}}+\beta_{4} \\
& \text { GENDER }_{\mathrm{ik}}+\beta_{5} \mathrm{AGE}_{\mathrm{ik} k} \\
& \quad+v_{\mathrm{k}}+\mu_{\mathrm{ik}}+\epsilon_{\mathrm{ijk}}
\end{aligned}
$$

The $\beta$ s are the fixed regression coefficients; error terms reflect residual variation at neighbourhood $\left(v_{\mathrm{k}}\right)$, individual $\left(\mu_{\mathrm{jk}}\right)$, and assessment $\left(\epsilon_{\mathrm{ijk}}\right)$ levels. Intraclass correlation coefficients were calculated to permit estimation of variance at the neighbourhood level as a percentage of total variance in problem scores over all three levels, in a model with no predictors.

Separate models were estimated for the six problem scores: CBCL Total, Externalising, and Internalising; YSR Total, Externalising, and Internalising. We also investigated whether the effect of NSD varied according to child AGE or GENDER, or as a function of EDUC or OCC. Because none of these interactions were statistically significant, they were excluded from the final models.

The model used to analyse neighbourhood influences on longitudinal changes in behavioural problems had only two levels, as the dependent variable was problem score at T2. Here, Tl problem score was included as a covariate, thus effectively assessing change in the dependent variable between $\mathrm{Tl}$ and T2. Analyses were similar to those above, with child AGE, GENDER, EDUC, and OCC (in addition to Tl problems) as level 1 predictors and NSD as level 2 predictor. Again, six separate models were estimated. Interaction effects between NSD and gender and between NSD and family SES measures were not significant and were therefore excluded from the final models.

\section{RESULTS}

\section{Sample characteristics}

Children's average age was 10.9 years at $\mathrm{Tl}(\mathrm{SD}=0.4$; range $10-12)$ and 13.1 years at $\mathrm{T} 2(\mathrm{SD}=0.5$; range $12-14)$. At both 


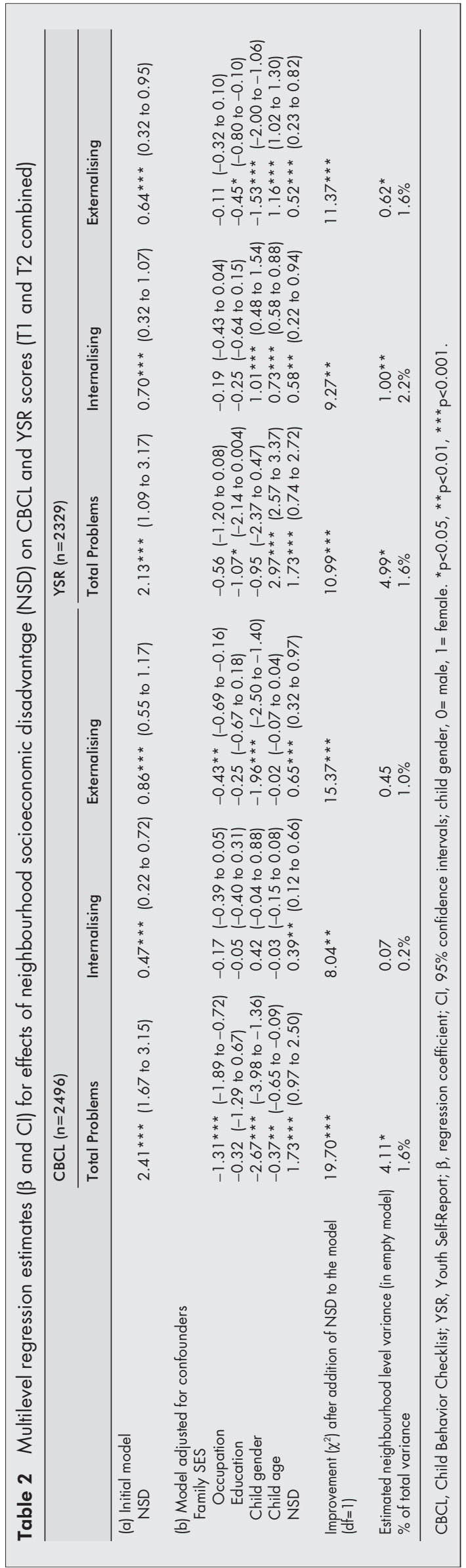

\section{Key points}

- Neighbourhood socioeconomic disadvantage was associated with greater behavioural problems in children, irrespective of family socioeconomic status.

- Living in a disadvantaged neighbourhood may exacerbate problems as children move from childhood into adolescence.

assessments, $51 \%$ were boys; over $90 \%$ were born in the Netherlands. Respondents came from 68 of the 74 Rotterdam neighbourhoods. At Tl, the median number of respondents per neighbourhood was 23 (range 1-155), with 40 neighbourhoods represented by more than 20 respondents.

At $\mathrm{Tl}, 73.4 \%(\mathrm{n}=2587)$ of the eligible cohort entered the study; at $\mathrm{T} 271.0 \%(\mathrm{n}=1836)$ of the $\mathrm{Tl}$ subjects participated again. To assess selective attrition from $\mathrm{T} 1$ to T2, we compared drop outs with completers with respect to gender, family SES, and behavioural problems at T1. Drop outs did not differ from T2 completers with respect to gender $\left(\chi^{2}=0.018 ; \mathrm{p}=0.894\right)$, but parental occupation $(t=6.204, \mathrm{df}=2226 ; \mathrm{p}<0.001)$ and education $(t=4.76, \mathrm{df}=1919 ; \mathrm{p}<0.001)$ were lower in drop outs. They scored significantly higher than T2 completers on T1 CBCL Total Problems (21.71 v 19.61; $t=3.12$, df=2494; $\mathrm{p}=0.002)$, but not on YSR Total Problems (26.32 v 25.08; $t=1.44, \mathrm{df}=2327 ; \mathrm{p}=0.151$ ).

\section{Problem scores}

Table 1 shows the descriptive statistics for CBCL and YSR measures at $\mathrm{T} 1$ and $\mathrm{T} 2$.

CBCL and YSR measures were modestly correlated. At T1, Pearson correlations were $0.44,0.44$, and 0.40 for Total, Externalising, and Internalising problems, respectively; at T2, these correlations were $0.39,0.38$, and 0.40 (all p values $<0.001$ ).

\section{Policy implications}

- Public health interventions to improve child mental health must take the neighbourhood environment into account.

\section{Neighbourhood effects on child problems}

As figure 1 shows, children living in disadvantaged neighbourhoods appeared to have more behavioural problems. Results of the multilevel analysis of combined T1 and T2 data, unadjusted for individual level effects, confirmed that NSD had significant effects on CBCL and YSR Total, Internalising, and Externalising problems over the age range 10-14 years (table 2, section a).

NSD and family SES were modestly correlated (NSD with education: $r=-0.25, \mathrm{p}<0.01$; NSD with occupation: $r=-0.31$, $\mathrm{p}<0.01$ ), with lower SES families tending to live in higher NSD neighbourhoods. We therefore extended the multilevel model with individual level indicators of family SES, also controlling for effects of child age and gender. Higher parental occupation was associated with fewer CBCL Total and Externalising problems, and higher parental education was associated with fewer YSR Total and Externalising problems (see table 2, section b). Parents reported fewer Total and Externalising problems in girls than in boys. On the YSR, girls reported more Internalising and boys more Externalising problems. Self reported problems increased with age, whereas parent reported problems were the same or even lower (Total Problems) for older children. Even after adjustment for the individual level variables, higher NSD was associated with more behavioural and emotional problems.

Neighbourhood level variance in problem scores was low, both in absolute terms and as a percentage of total variance in 
Table 3 Multilevel regression estimates ( $\beta$ and $C l$ ) for effects of NSD on changes in Total Problems from T1 to T2

\begin{tabular}{|c|c|c|}
\hline & \multicolumn{2}{|l|}{ Total Problems (T2) } \\
\hline & $\mathrm{CBCL}(n=1565)$ & YSR $(n=1787)$ \\
\hline \multicolumn{3}{|l|}{ (a) Model adjusted for baseline problem scores only } \\
\hline Total Problems (T1) & $0.67^{* * *}(0.63$ to 0.71$)$ & $0.47^{* * *}(0.42$ to 0.52$)$ \\
\hline NSD & $0.82 *(0.04$ to 1.59$)$ & $1.18 *(0.18$ to 2.17$)$ \\
\hline Improvement $\left(\chi^{2}\right)$ after NSD added to the model $(d f=1)$ & $4.42^{*}$ & $5.37^{\star}$ \\
\hline \multicolumn{3}{|l|}{ (b) Model adjusted for baseline scores and confounders } \\
\hline Total Problems (T1) & $0.66^{* * *}(0.62$ to 0.71$)$ & $0.49 * * *(0.44$ to 0.53$)$ \\
\hline \multicolumn{3}{|l|}{ Family SES } \\
\hline Occupation & $0.07(-0.54$ to 0.68$)$ & $1.01 * *(0.28$ to 1.73$)$ \\
\hline Education & $-0.42(-1.44$ to 0.60$)$ & $-1.90 * * *(-3.12$ to -0.67$)$ \\
\hline Child gender & $0.17(-1.16$ to 1.50$)$ & $4.99 * * * \quad(3.37$ to 6.62$)$ \\
\hline Child age (T2) & $0.21(-1.20$ to 1.62$)$ & $0.79(-1.02$ to 2.61$)$ \\
\hline NSD & $0.75\left(^{*}\right)(-0.05$ to 1.55$)$ & $1.05 *(0.03$ to 2.08$)$ \\
\hline Improvement $\left(\chi^{2}\right)$ after NSD added to the model $(d f=1)$ & $3.40\left(^{*}\right)$ & $4.08^{*}$ \\
\hline Estimated neighbourhood level variance (in empty model) & 1.37 & 0 \\
\hline$\%$ of total variance & $0.6 \%$ & $0 \%$ \\
\hline
\end{tabular}

a model with no predictors. Nevertheless, neighbourhood variance was statistically significant for four of the six behavioural outcomes.

\section{Neighbourhood effects on changes in behavioural problems over time}

Table 3 summarises results of models examining the effect of NSD on YSR and CBCL changes over the two year follow up, with Total Problems at T2, controlling for T1 levels, as the outcome measure.

$\mathrm{Tl}$ problems were a very significant predictor of $\mathrm{T} 2$ problems, indicating considerable continuity of behavioural problems from late childhood into early adolescence. A model with NSD as the only additional predictor (table 3, section a) showed that children in high NSD neighbourhoods developed significantly more Total Problems (both parent and self reported) over the follow up period. After controlling for family SES, child's age and gender, in the adjusted models (table 3 , section $\mathrm{b}$ ), the association between NSD and behavioural problems remained statistically significant for YSR scores; for CBCL scores, a trend in the same direction was observed.

Family SES effects on YSR problems were in the expected direction for parental education, with higher education associated with smaller increases in self reported problems; surprisingly, higher parental occupation was associated with a greater increase in problems. Girls reported significantly greater increases in Total Problems over the follow up period than boys, attributable mainly to an increase in Internalising scores. Changes in CBCL scores showed no clear gender differences. In contrast with Total Problems, models with Internalising or Externalising scores as dependent variable (data not presented) showed no clear effects of NSD after inclusion of control variables.

\section{DISCUSSION}

NSD was associated with child and parent reported behavioural problems, over and above the effect of individual level SES. Furthermore, longitudinal results suggested that NSD may lead to an increase in problem occurrence from late childhood into early adolescence. We found no evidence that NSD effects differed in magnitude for children from high compared with low SES families, or for boys compared with girls. Living in a disadvantaged neighbourhood thus represents an independent risk factor for children. Data that might shed light on the pathways through which NSD may have influenced behaviour were not available. Possible mechanisms include perceived danger (which can lead to anxiety in children and coercive parenting styles), exposure to inappropriate peers and adult role models, and low levels of neighbourhood cohesion, informal social control, and collective efficacy. ${ }^{1-6} 914$

These results replicate and extend a number of findings concerning neighbourhood effects on mental health in children and adolescents. Kalff et al demonstrated an association between moderate levels of NSD and child behavioural problems, using CBCL data from 5-7 year olds in a small Dutch city. ${ }^{15}$ In the same city NSD was associated with behavioural problems in 11 year olds. ${ }^{14}$ We have shown that these findings can be generalised to older children (10-14 years) in a much larger, urban setting. Despite differences in national social welfare policies, neighbourhood effects on child development of similar magnitude are likely to exist in other large European cities; comparative studies are needed to clarify effects of more extreme or concentrated NSD in other countries. Exclusion of children with non-Dutch nationality may have lead to an underestimate of neighbourhood effects, because of the overrepresentation of recent immigrants in neighbourhoods with higher NSD. Data for the current analysis were collected 10 to 13 years ago; although sociodemographic changes have occurred in Rotterdam, including a slight increase in the percentage of immigrants, neighbourhood income data show no clear evidence for an increasing (or decreasing) gradient in NSD.*

By examining Internalising and Externalising clusters as well as Total Problems, this study provides evidence that NSD is associated with a wide range of emotional and behavioural problems. Although parent reports (CBCL) showed a somewhat stronger effect of NSD on Externalising than on Internalising scores, both clusters showed similar associations with NSD when YSR scores were the outcome measures. This suggests that NSD could play a part in the development of depression as well as conduct disorders.

This study appears to be the first to use longitudinal data to investigate neighbourhood effects on changes in children's

* Mean disposable income per inhabitant in the five poorest neighbourhoods was $44 \%$ of that in the five wealthiest neighbourhoods in 1995, and $49 \%$ in 2001 (Dutch Central Bureau of Statistics). 
behavioural problems over time. As hypothesised, children living in more disadvantaged neighbourhoods reported greater increases in Total Problems over the two year follow up period; parents indicated a trend in the same direction. Although estimated effects of NSD on changes in problem behaviour over time were fairly small, it is important to recall that overall increases in self reported behavioural problems over the age range investigated were also small; parents actually reported a slight decrease in Total Problems. After adjustment for Tl levels there was thus little variability in T2 outcome measures left to explain. NSD effects may have become even more evident over a longer follow up, as previous studies indicate that self reported problems increase throughout adolescence. ${ }^{13}$

Additional strengths of this study include the large community sample, representing almost all neighbourhoods of a single metropolitan area. NSD, therefore, had a wide range and a continuous distribution. This measure was calculated from city administrative records and cannot therefore have been biased by participants' perceptions of neighbourhood quality. The number of sampled neighbourhoods $(n=68)$ and number of participants per neighbourhood were comparatively large, an important advantage in multilevel analysis. ${ }^{7}$

The study has some limitations. In particular, low SES families were somewhat underrepresented. At Tl parental occupation and education were lower and YSR Total Problems higher in participants who failed to complete sufficient questionnaire items for inclusion. ${ }^{10}$ Similar forms of selective attrition appeared to have operated between $\mathrm{T} 1$ and T2; drop outs were more likely to have come from lower SES families and also had significantly higher CBCL total problem scores at Tl than completers. To assess whether attrition biased the main findings, we examined whether later drop outs showed a different pattern than completers in the relation between NSD and problems at Tl. Multilevel estimates of the interaction between NSD and later drop out status on T1 Total Problems (either CBCL or YSR) were not, however, statistically significant.

Although the multilevel analytical approach takes into account the similarity of subjects within a neighbourhood, some part of the estimated effect of NSD in the current models might be attributable to individual level effects, if families with certain unmeasured characteristics were more likely to reside in disadvantaged than in advantaged neighbourhoods. ${ }^{4}$ Family characteristics previously reported to have an association with child behaviour measures, such as parental marital status and single parent household, ${ }^{14}{ }^{15}$ were not included in this analysis, although these characteristics could plausibly be clustered within neighbourhoods. Even if individual level SES effects are fully controlled, it remains difficult to determine conclusively whether associations between NSD and problem behaviours are actually caused by neighbourhood characteristics or instead reflect a selection bias in how families come to live in disadvantaged neighbourhoods. ${ }^{6}$ Experimental designs in which randomly selected families are offered incentives to move to more affluent neighbourhoods ${ }^{16}$ and genetic designs that can tease true environmental effects apart from genetic liability to behavioural problems ${ }^{17}$ are two approaches that can help differentiate causal from selection processes. Our finding that NSD was associated with increasing problems in children over follow up lends support to the causal interpretation.

As in previous studies, estimated effects of NSD were modest and neighbourhood level variance low. ${ }^{7}$ However, the widespread occurrence of disadvantaged neighbourhoods suggests that the public health consequences of growing up in such contexts will be large. Community interactions aimed at bettering neighbourhood conditions are therefore likely to have considerable impact.

\section{Authors' affiliations}

J Schneiders, M Drukker, J van Os, N A Nicolson, Department of Psychiatry and Neuropsychology, South Limberg Mental Health Research Network, EURON, Maastricht University, Netherlands

J van der Ende, F C Verhulst, Department of Child and Adolescent

Psychiatry, Erasmus Medical Centre-Sophia Children's Hospital

Rotterdam, Netherlands

\section{REFERENCES}

1 Aneshensel CS, Sucoff CA. The neighborhood context of adolescent mental health. J Health Soc Behav 1996;37:293-310.

2 Gephart MA. Neighborhoods and communities as contexts for development. In: Brooks-Gunn J, Duncan GJ, Aber JL, eds. Neighborhood poverty. New York: Russell Sage Foundation, 1997: 1-43.

3 Ingoldsby EM, Shaw DS. Neighborhood contextual factors and early-starting antisocial pathways. Clin Child Fam Psychol Rev 2002;5:21-55.

4 Leventhal T, Brooks-Gunn J. The neighborhoods they live in: the effects of neighborhood residence on child and adolescent outcomes. Psychol Bull 2000;126:309-37.

5 McLoyd VC. Socioeconomic disadvantage and child development. Am Psychol 1998;53:185-204.

6 Sampson RJ, Morenoff JD, Gannon-Rowley T. Assessing "neighborhood effects": social processes and new directions in research. Annu Rev Sociol 2002;28:443-78.

7 Diez-Roux AV. Multilevel analysis in public health research. Annu Rev Public Health 2000;21:171-92.

8 Laitinen-Krispijn S, Van der Ende J, Hazebroek-Kampschreur AA, et al. Pubertal maturation and the development of behavioural and emotional problems in early adolescence. Acta Psychiatr Scand 1999;99:16-25.

9 Boyle MH, Lipman EL. Do places matter? Socioeconomic disadvantage and behavioral problems of children in Canada. J Consult Clin Psychol 2002;70:378-89.

10 van Hoeken D. Pubertal status and problem behavior: an epidemiological study of 11 -year-olds. [PhD dissertation.] Rotterdam: Erasmus University, 1994.

11 Achenbach TM, Edelbrock CS. Manual for the child behavior checklist and revised profile. Burlington, VT: University of Vermont, Department of Psychiatry, 1983.

12 Achenbach TM, Edelbrock CS. Manual for the youth self-report and profile. Burlington, VT: University of Vermont, Department of Psychiatry, 1987.

13 Verhulst FC, van der Ende J. Agreement between parents' reports and adolescents' self-reports of problem behavior. J Child Psychol Psychiatry 1992;33:1011-23.

14 Drukker M, Kaplan CD, Feron FJM, et al. Children's health-related quality of life, neighbourhood socio-economic deprivation and social capital. A contextual analysis. Soc Sci Med 2003;57:825-41.

15 Kalff AC, Kroes M, Vles JS, et al. Neighbourhood level and individual level SES effects on child problem behaviour: a multilevel analysis. J Epidemiol Community Health $2001 ; 55: 246-50$.

16 Katz LF, Kling J, Liebman JB. Moving to opportunity in Boston: early results of a randomized mobility experiment. Quarterly Journal of Economics $2001 ; 116: 606-54$

17 Caspi A, Taylor A, Moffitt TE, et al. Neighborhood deprivation affects children's mental health: environmental risks identified in a genetic design. Psychol Sci 2000;1 1:338-42. 